\title{
The Impact of COVID-19 on Mental Health: Psychosocial Conditions of Students with and without Special Educational Needs
}

\author{
David Scheer $^{1, *(\mathbb{D})}$ and Désirée Laubenstein ${ }^{2}$ \\ 1 Faculty for Special Needs Education, Ludwigsburg University of Education, 71634 Ludwigsburg, Germany \\ 2 Institute for Educational Science, Paderborn University, 33098 Paderborn, Germany; \\ desiree.laubenstein@upb.de \\ * Correspondence: david.scheer@ph-ludwigsburg.de
}

Citation: Scheer, David, and Désirée Laubenstein. 2021. The Impact of COVID-19 on Mental Health: Psychosocial Conditions of Students with and without Special Educational Needs. Social Sciences 10: 405. https://doi.org/10.3390/socsci10110405

Academic Editor: Nigel Parton

Received: 20 September 2021

Accepted: 14 October 2021

Published: 20 October 2021

Publisher's Note: MDPI stays neutral with regard to jurisdictional claims in published maps and institutional affiliations.

Copyright: (c) 2021 by the authors. Licensee MDPI, Basel, Switzerland. This article is an open access article distributed under the terms and conditions of the Creative Commons Attribution (CC BY) license (https:// creativecommons.org/licenses/by/ $4.0 /)$.

\begin{abstract}
Given the pandemic-induced school lockdown in Germany in the spring of 2020, COVID-19 evidently had a negative impact on child and adolescent mental health and wellbeing. However, there is no evidence regarding the specific problems of students with special educational needs in emotional and behavioral disorders (E/BD) during or after the school lockdown. Thus, this study bridges the gap. A sample of 173 students across Germany was included in the analysis. The students were rated by their teachers in an online survey via a standardized teacher-report form for emotional and behavioral problems and competencies, as well as perceptions of inclusion. Several student- and teacher-level predictors were applied in a stepwise regression analysis. The results showed that the school lockdown marginally impacted E/BD, with small differences between student groups. The strongest predicting variable was students' psychosocial situation. Hence, the psychosocial situation of students should be monitored by teachers and school psychologists to provide sufficient support during lockdown.
\end{abstract}

Keywords: COVID-19; lockdown; externalizing problems; internalizing problems; positive schoolrelated behavior

\section{Introduction}

During the COVID-19 pandemic, schools in Germany canceled in-person teaching in March 2020. The lockdown was eased systematically following the Easter break in April, and, at least since the end of the summer break, schools have completely re-opened. Research has shown the impact of the pandemic situation on children's and adolescents' wellbeing and mental health. Although the research base is small for students with special educational needs (SEN) in the area of emotional and behavioral disorders (E/BD), the negative impacts of school closures can also be assumed for them.

\subsection{Special Educational Needs and Emotional and Behavioral Disorders}

Since the current study was conducted in Germany, it is important to introduce the terminology used for SEN and E/BD. According to the Standing Conference of the Ministers of Education and Cultural Affairs of the Länder in the Federal Republic of Germany [Kultusministerkonferenz], which is responsible for coordinating the school system across the sixteen federal states in Germany, SEN students are defined as children and young people who either have disabilities or face the threat of disablement (Kultusministerkonferenz 2019, p. 251). SEN is used as an administrational category to allocate and legitimize resources for individualized remedial support or special accommodations, either in regular classrooms or in special schools. Since there are no general guidelines for SEN diagnoses across Germany, regulations for diagnosing SEN (and therefore the number of SEN students) vary across the federal states (Scheer and Melzer 2020). 
The term "special educational needs in emotional and behavioral disorders" (SENE/BD) describes "a particularly severe education problem caused by particularly pronounced emotional-social developmental and behavioral disorder or characterized by an accumulation of severe risk factors for development" (Blumenthal et al. 2020, pp. 12-13). Following this definition, SEN-E/BD refers to the international term of emotional and behavioral disorders (E/BD) (see Forness and Kavale 2000), which differentiates between externalizing problems (EP; for example, aggressive behavior, defiant and oppositional behavior, and hyperactivity) and internalizing problems (IP; for example, anxiety disorders or depression).

In the school year 2018-2019, 1.3\% of all students in Germany were diagnosed as SEN-E/BD, and $0.56 \%$ of all students were attending a special school for SEN-E/BD (Scheer and Melzer 2020). Whereas, studies on child and adolescent mental health indicated a prevalence of E/BD between 16\% and 21\% (Baumgarten et al. 2018).

\subsection{Effects of the Pandemic Situation on Children and Adolescents}

In a rapid umbrella review of several previous reports, Baumann (2020) summarized evidence of mental health and psychosocial problems among children and adolescents due to quarantine measures during the pandemic (Brooks et al. 2020; Graber et al. 2020; Henssler et al. 2020; Hossain et al. 2020; Imran et al. 2020; Röhr et al. 2020; Sharan and Rajhans 2020). The consequences of quarantine measures seemed to correspond with the socioeconomic situation of families. Although quarantine conditions are stricter than Germany's overall lockdown measures, the latter may have similar effects (Chawla et al. 2021; Lee 2020; Sharma et al. 2020; Ron and Cuéllar-Flores 2020; Singh et al. 2020). Despite acknowledging the possible negative effects of school lockdowns on mental health, Chawla et al. (2021) assumed that the lockdown could mean an absence of bullying or school pressure for SEN students; thus, it might have a somewhat positive effect on their psychological wellbeing. It was found that parental stress during the lockdown may be transferred to children, where the lockdown situation might induce higher in-family violence, emotional neglect, or isolation (Clemens et al. 2020; Fontanesi et al. 2020; Mechili et al. 2021; Spinelli et al. 2020). This further underpins the assumption of the pandemic's negative impact on child wellbeing. Furthermore, the pandemic challenged the system of public child welfare. Moreover, although child welfare practitioners appear to have adjusted to the new situation, the long-term effects of the lockdown on the public child welfare system remain unknown (Baginsky and Manthorpe 2021; Jentsch and Schnock 2020).

Empirical research shows that the pandemic induced higher psychological and mental health issues for students (Chen et al. 2020; Idoiaga Mondragon et al. 2021; Ravens-Sieberer et al. 2020, 2021; Xie et al. 2020; Langmeyer et al. 2020; Pearcey et al. 2020). Although Pearcey et al. (2020) reported an increase in primary school-aged children's emotional, behavioral, and attentional challenges from a parent-carer perspective, they found a reduction in such problems for secondary school-aged children or adolescents and those with SEN or pre-existing mental health issues. Further, they found no changes regarding adolescents self-reported difficulties, whereas, an increase in behavioral difficulties was reported for high-income households. Langmeyer et al. (2020) found that about a third of parents reported problems in coping with the lockdown, which was correlated with conflicts and chaos within the family. They also found that more children seemed to suffer from loneliness in low-income households. However, according to Langmeyer et al. (2020), children's constant or frequent contact with teachers or kindergarten educators had a protective effect on their mental health.

Research on schooling during the lockdown in Germany shows that distance teaching and learning, and parental support for learning, were challenging for all those involved (Huber et al. 2020; Wacker et al. 2020; Wildemann and Hosenfeld 2020). Although students acknowledged more flexibility and individualization of learning, they also lacked communication with and feedback from teachers (Wacker et al. 2020). Children and adoles- 
cents with lower socio-economic status faced greater challenges with distance learning or homeschooling (Wildemann and Hosenfeld 2020).

Casale et al. (2020a) found that most German federal states had no further regulations and documentation about the continuation of special education services for SEN students during the school lockdown. In an online survey, SEN teachers analyzed the actual implementation of and obstacles and conditions for SEN support and digital learning during the school lockdown (Börnert-Ringleb et al. 2021; Casale et al. 2020b).

However, from an international and German perspective, prior studies present insufficient empirical evidence on (1) the specific effects of the pandemic school lockdown on SEN students with emotional and behavioral disorders (E/BDs) and (2) predictors for such effects from the teachers' perspective.

\section{Research Questions}

Therefore, this study considers the following questions:

- (Q1) After the re-opening of schools, did E/BDs differ between students with and without SEN-E/BD?

- (Q2) Were there any differences in psychosocial threats and coping with distance learning between students with and without SEN-E/BD during the school lockdown?

- (Q3a) Did teachers perceive changes in their students' E/BD after lockdown?

- $\quad(\mathrm{Q} 3 b)$ Which student-teacher-level predictors affected these changes?

\section{Methods}

\subsection{Procedure}

The present study employed a retrospective cross-sectional design to investigate teachers' perceptions of changes in students' E/BD. Teachers from across Germany were invited to participate in an online survey. The invitation was advertised on social media (Twitter and Facebook), mailing lists of teacher associations and unions, and press releases. Two federal states (Baden-Wuerttemberg and Rhineland-Palatinate) gave explicit permission to contact schools officially. The survey was conducted using LimeSurvey Version 3.17.16 (LimeSurvey 2019), and hosted by the second author's university. Before the survey, participants were provided information about the study to grant conclusive informed consent for participation. Therefore, given the ad-hoc sample, we could not determine our sample size a priori.

In the first part of the survey, participants were required to disclose the schools in which they taught, the number of students in each class, and how many students were diagnosed with SEN-E/BD. They were also required to state the number of students they were willing to assess during the survey (two, three, four, or five). Figure 1 illustrates how scenarios are chosen from the answers provided in this part, which determined the next step for participants and the randomization procedure characteristics. The instructions that participants were given in each of the four scenarios displayed in Figure 1 can be found in Appendix A (Appendices A.1-A.4). The full structure of the survey is provided via OSF as a LimeSurvey structure file ( $\left.{ }^{*} .1 s s\right)$ and as an Excel spreadsheet to make the procedure replicable.

After completing the questionnaire (measurements described below), participants were asked if they were willing to (a) participate in a repeated survey in case of new lockdowns, and (b) participate in qualitative interviews for further in-depth insights (qualitative interviews with 22 teachers have been conducted in early 2021 and are currently being analyzed). 


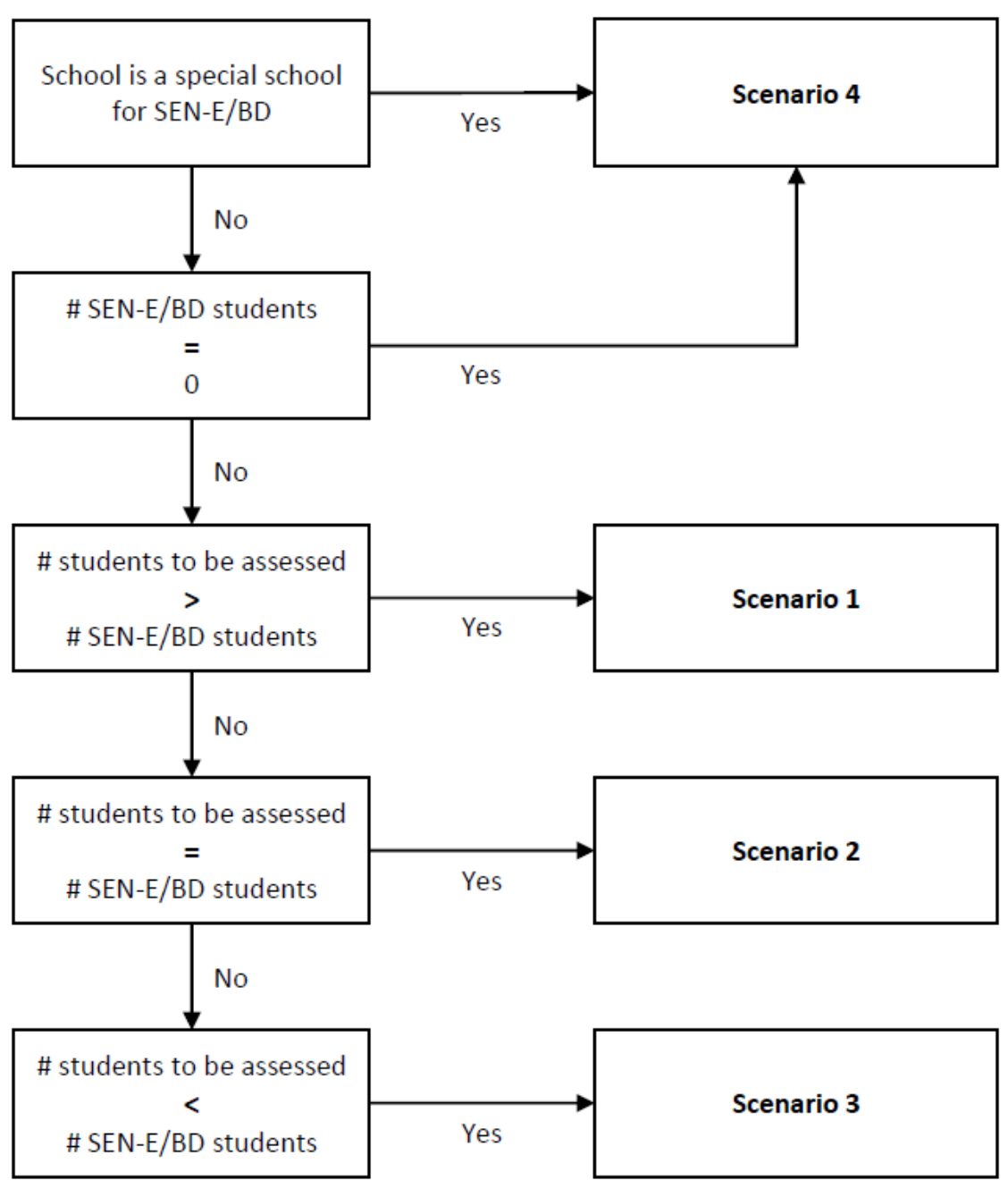

Figure 1. Path diagram to determine the correct scenario of the questionnaire.

\subsection{Measurements}

In this paper, we only report those variables that were included in the data analysis. The full set of variables measured can be obtained from the dataset and its documentation on OSF.

The scale ranges are transformed as per their 0 starting point, except for the retrospective scales which range from -2 to +2 (see Section 3.2.3). Due to technical reasons, scale ranges (answer codes) in the questionnaire deviate from this structure.

\subsubsection{Students' Sociodemographic Data}

Teachers were required to provide students' gender, grade, age, and living environment conditions. Further, they provided information on any official diagnosis of SEN.

\subsubsection{DBR-PUTSIE}

To assess students' internalizing problems (IP), externalizing problems (EP), and positive school-related behavior (P-SRB), we employed the DBR-PUTSIE (Schurig et al. 2020), a standardized instrument for directly assessing behavior in schools. It is published under a CC BY-NC-SA 4.0 license and is free to use for research purposes. The DBR-PUTSIE is based on well-established instruments, such as the Strengths and Difficulties Questionnaire (SDQ) (Goodman 2005, 1999), and on the behaviors described by the Diagnostic and Statistical Manual of Mental Disorders, fifth edition (DSM-5). Development of the DBR-PUTSIE is strongly related to that of the Direct Behavior Rating and Multi-Item 
Scales by Gebhardt et al. (2018). Thus, we assumed comparable psychometric properties (Gebhardt et al. 2019). The DBR-PUTSIE covers the three dimensions of EP (oppositional defiant behavior (4 items), inattention (6 items), impulsiveness (6 items)), IP (emotional problems (5 items), peer problems (3 items)), and P-SRB (5 items). The items are rated on a seven-point scale with verbalized endpoints $(1=$ never, $7=$ always $)$.

The DBR-PUTSIE was preferred over the SDQ because a seven-point rating scale is preferable for direct behavior ratings (Christ et al. 2009). Furthermore, the SDQ is not licensed for online survey use.

The items are displayed in Appendix B Table A1. We observed acceptable to good internal consistency, with $\alpha=0.85$ to 0.95 , and a corrected item-total correlation of $r_{\text {it }}>0.5$ for all items, with only five items falling below an $r_{\text {it }}$ of 0.6 .

\subsubsection{Retrospective Ratings}

After participants assessed the current state of social/emotional development with the original DBR-PUTSIE instructions, they were asked to assess the current situation relative to the situation before school lockdown on a fully verbalized five-point scale $(-2=$ much lower than before, $-1=$ somewhat lower than before, $0=$ no change, $+1=$ somewhat higher than before, $+2=$ much higher than before). As shown in Appendix B Table A2, the internal consistency ranged from 0.82 to 0.92 . The corrected item-total correlation was slightly lower than for the rating of the current situation, with two items falling below an $r_{\text {it }}$ of 0.5 . Four items showed an $r_{\text {it }}$ between 0.5 and 0.6.

\subsubsection{Students' Coping with Distance Learning and Psychosocial Threats during the Lockdown}

A scale measuring how students addressed distance learning during school lockdown ("coping with distance learning", 6 items) was developed following Huber et al. (2020). The items were rated on a fully verbalized five-point scale ( $0=$ not true at all, $1=$ somewhat not true, 2 = yes and no, 3 = somewhat true, 4 = very true). Along with the scale on students coping with distance learning, five items on psychosocial threats were presented to be assessed on the same rating scale. Table A3 in the Appendix B indicates acceptable internal consistency for both scales ( $\alpha=0.83$ and 0.81 , respectively). For the scale on coping with distance learning, one item had a poor $r_{\text {it }}$ of 0.256 , one item had a good $r_{\text {it }}$ of 0.551 , and the other items were all above 0.7 . For the scale on psychosocial threats, one item had an $r_{\text {it }}=0.365$, while all other items were above 0.6 .

\subsubsection{Teacher Variables}

Using a five-point rating scale ( 1 = never, 5 = almost always), we asked teachers how often they employed analogue and digital teaching or instruction for distance teaching during the school lockdown. The two questions were taken from the SOLVE-questionnaire (Casale et al. 2020b) in Börnert-Ringleb et al. (2021).

Using the SOLVE-questionnaire, we employed a scale to measure the teachers' perception of their student-teacher relationship following Pianta et al. (2008). The scale consists of nine items to be rated on a fully verbalized five-point scale ( $0=$ not true at all, $1=$ somewhat not true, 2 = yes and no, 3 = somewhat true, 4 = very true). Since the original scale has yet to be published, we cannot provide the full text of the items here. The internal consistency was acceptable $(\alpha=0.78)$. Three items showed an $r_{\text {it }}<0.5$.

\subsection{Data Inclusion and Data Availability}

Only complete cases were included in the data analysis. The raw data from LimeSurvey, the final data set used for analysis, and the $\mathrm{R}$ input are available via OSF.

\subsection{Participants}

In total, 94 teachers (28 from primary schools, 37 from secondary schools (including comprehensive schools, academic high schools, and vocational schools), and 29 from special 
schools) participated in the survey. The average number of students (with SEN-E/BD and total) in the participants' classes is displayed in Table 1 . From the secondary schools, 2 participants were from a lower secondary school, 3 from a middle secondary school, 6 from an integrated secondary school, 12 from an academic high school, 9 from a comprehensive school, and 5 from a vocational school.

Data were included from 173 (55 female) students, of which 82 (11 female) were officially diagnosed with SEN-E/BD, and 27 (10 female) with other types of SEN. Table 2 presents a summary of the students' sociodemographic data. It was observed that students with SEN-E/BD were more likely to be living in foster families or youth welfare facilities than students without SEN-E/BD.

Table 1. Teachers participating in the survey.

\begin{tabular}{ccccc}
\hline & Overall & Primary School & Secondary School & Special School \\
\hline N & 94 & 28 & 37 & 29 \\
Students in class (mean (SD)) & $19.41(8.82)$ & $20.64(3.95)$ & $25.59(8.26)$ & $10.34(4.28)$ \\
Students in class with SEN-E/BD (mean (SD)) & $2.44(4.19)$ & $1.25(1.94)$ & $1.56(3.06)$ & $6.50(6.48)$ \\
\hline
\end{tabular}

Note. SEN represents "Special Educational Needs", E/BD represents "Emotional and Behavioral Disorders".

Table 2. Students' sociodemographic data.

\begin{tabular}{|c|c|c|c|c|c|}
\hline & Overall & SEN-E/BD & Other SEN & No SEN & $p$ \\
\hline $\mathrm{N}$ & 173 & 82 & 27 & 64 & \\
\hline Gender $=$ female $(\%)$ & $55(31.8)$ & $11(13.4)$ & $10(37.0)$ & $34(53.1)$ & $<0.001$ \\
\hline Age (mean (SD)) & $12.09(3.74)$ & $11.89(2.62)$ & $13.89(6.15)$ & $11.58(3.47)$ & 0.021 \\
\hline Grade (mean (SD)) & $6.23(3.02)$ & $6.07(2.64)$ & $7.04(3.31)$ & $6.09(3.32)$ & 0.321 \\
\hline Living environment (\%) & & & & & 0.005 \\
\hline Parents/family of origin & $148(85.5)$ & $62(75.6)$ & $25(92.6)$ & $61(95.3)$ & \\
\hline Foster family & $4(2.3)$ & $4(4.9)$ & $0(0.0)$ & $0(0.0)$ & \\
\hline Children's home/group accommodation facilities & $14(8.1)$ & $13(15.9)$ & $1(3.7)$ & $0(0.0)$ & \\
\hline Other & $52.9)$ & $1(1.2)$ & $1(3.7)$ & $3(4.7)$ & \\
\hline Missing & $2(1.2)$ & $2(2.4)$ & $0(0.0)$ & $0(0.0)$ & \\
\hline
\end{tabular}

\subsection{Data Analysis}

Data analyses were performed in the R language ( $R$ Core Team 2020), using the R Studio Environment (RStudio 2020). Descriptive statistics were analyzed using the describe() and describeBy() commands from the package "psych" (Revelle 2020).

In the first step of the analysis, we compared social and emotional development, coping with distance learning, and psychosocial threats during lockdown across SEN-E/BD students, other SEN students, and students without SEN. One-way ANOVAs were computed using the $\operatorname{lm}()$-function along with the apa.1way.table()-function from "apaTables" (Stanley 2018).

Predictors for social and emotional problems and the effect of school lockdown were analyzed using stepwise regression analysis. Hence, we incorporated students' SEN status as predictors in block one (Step 1). Students' coping with distance learning and psychosocial threats during lockdown were added in Step 2. Step 3 included the teachers' perception of the student-teacher relationship. Stepwise regression was conducted for each dependent variable (effects of the lockdown on EP, IP, and P-SRB), using the $1 \mathrm{~m}()$ command from the base functions of $\mathrm{R}$ and the apa.reg.table() function from "apaTables".

\section{Results}

Table 3 shows the descriptive statistics for EP, IP, P-SRB, psychosocial threats, and students' coping with distance learning. The changes in EP, IP, and P-SRB that teachers reported as an effect of the lockdown were rather marginal. However, students with SEN seemed to show a higher increase in EP than students without SEN. Furthermore, students 
with SEN seemed to show an increase in IP during lockdown, while students without SEN showed a decrease in IP. Additionally, teachers reported similar decreases in P-SRB in all groups.

Table 3. Descriptive statistics categorized by SEN.

\begin{tabular}{|c|c|c|c|c|c|c|c|c|c|}
\hline & \multicolumn{3}{|c|}{ SEN-E/BD } & \multicolumn{3}{|c|}{ Other SEN } & \multicolumn{3}{|c|}{ No SEN } \\
\hline & $\mathbf{M}$ & SD & SE & $\mathbf{M}$ & SD & SE & $\mathbf{M}$ & SD & SE \\
\hline \multicolumn{10}{|c|}{ Current Situation } \\
\hline School Related Behavior (P-SRB) & 2.58 & 1.15 & 0.1270 & 3.30 & 1.51 & 0.2897 & 4.07 & 1.45 & 0.1812 \\
\hline Externalizing Problems (EP) & 3.30 & 1.08 & 0.1194 & 2.61 & 1.61 & 0.3095 & 1.48 & 1.15 & 0.1442 \\
\hline Internalizing Problems (IP) & 2.49 & 1.18 & 0.1301 & 1.77 & 1.05 & 0.2026 & 1.32 & 1.00 & 0.1245 \\
\hline \multicolumn{10}{|c|}{ Lockdown Situation } \\
\hline Psychosocial Threats & 0.92 & 0.85 & 0.0939 & 0.81 & 0.82 & 0.1580 & 0.56 & 0.66 & 0.0830 \\
\hline Coping with Distance Learning & 1.57 & 0.89 & 0.0984 & 1.78 & 0.89 & 0.1704 & 2.43 & 0.95 & 0.1192 \\
\hline \multicolumn{10}{|c|}{ Effect of Lockdown } \\
\hline School Related Behavior (P-SRB) & -0.16 & 0.50 & 0.0552 & -0.10 & 0.38 & 0.0727 & -0.12 & 0.56 & 0.0697 \\
\hline Externalizing Problems (EP) & 0.18 & 0.40 & 0.0441 & 0.14 & 0.36 & 0.0684 & 0.02 & 0.49 & 0.0614 \\
\hline Internalizing Problems (IP) & 0.09 & 0.34 & 0.0374 & 0.06 & 0.19 & 0.0368 & -0.08 & 0.41 & 0.0507 \\
\hline
\end{tabular}

\subsection{Current EP, IP, and P-SRB}

As shown in Table 4, students' current EP, IP, and P-SRB were significantly different across the groups, as were psychosocial threats and coping with distance learning during lockdown, with partial $\eta^{2}$ ranging from 0.20 (IP) up to 0.33 (EP). However, as displayed in Table 5, for current EP and P-SRB, all pairwise group comparisons were significant. For current IP, only students with SEN-E/BD differed significantly from each of the other groups.

Table 4. ANOVA with current situation (EP, IP, P-SRB) and lockdown situation (PST, CDL) as criteria.

\begin{tabular}{|c|c|c|c|c|c|c|c|}
\hline & SS & Df & MS & F & $p$ & Partial $\eta^{2}$ & $95 \%$ CI of $\eta^{2}$ \\
\hline \multicolumn{8}{|c|}{ Externalizing Problems (EP) } \\
\hline (Intercept) & 183.35 & 1 & 183.35 & 126.80 & $<0.001$ & & \\
\hline SEN & 119.50 & 2 & 59.75 & 41.32 & $<0.001$ & 0.33 & {$[0.21,0.42]$} \\
\hline Error & 245.82 & 170 & 1.45 & & & & \\
\hline \multicolumn{8}{|c|}{ Internalizing Problems (IP) } \\
\hline (Intercept) & 84.57 & 1 & 84.57 & 70.58 & $<0.001$ & & \\
\hline SEN & 49.96 & 2 & 24.98 & 20.85 & $<0.001$ & 0.20 & {$[0.10,0.29]$} \\
\hline Error & 203.72 & 170 & 1.20 & & & & \\
\hline \multicolumn{8}{|c|}{ School Related Behavior (P-SRB) } \\
\hline (Intercept) & 293.37 & 1 & 293.37 & 167.09 & $<0.001$ & & \\
\hline SEN & 79.77 & 2 & 39.88 & 22.72 & $<0.001$ & 0.21 & {$[0.11,0.31]$} \\
\hline Error & 298.48 & 170 & 1.76 & & & & \\
\hline \multicolumn{8}{|c|}{ Psychosocial Threats (PST) } \\
\hline (Intercept) & 17.93 & 1 & 17.93 & 29.35 & $<0.001$ & & \\
\hline SEN & 4.73 & 2 & 2.37 & 3.87 & 0.023 & 0.04 & {$[0.00,0.11]$} \\
\hline Error & 103.84 & 170 & 0.61 & & & & \\
\hline \multicolumn{8}{|c|}{ Coping with Distance Learning (CDL) } \\
\hline (Intercept) & 85.33 & 1 & 85.33 & 102.21 & $<0.001$ & & \\
\hline SEN & 27.09 & 2 & 13.54 & 16.23 & $<0.001$ & 0.16 & {$[0.07,0.25]$} \\
\hline Error & 141.93 & 170 & 0.83 & & & & \\
\hline
\end{tabular}


Table 5. Pairwise comparisons from ANOVA.

\begin{tabular}{|c|c|c|c|c|}
\hline \multirow[t]{2}{*}{ Comparison } & \multirow[t]{2}{*}{ Difference } & \multicolumn{2}{|c|}{$95 \%$ CI } & \multirow[t]{2}{*}{$p$} \\
\hline & & LL & UL & \\
\hline SEN-E/BD vs. Other SEN & 0.69 & 0.06 & 1.32 & 0.028 \\
\hline No SEN vs. Other SEN & -1.13 & -1.78 & -0.48 & $<0.000$ \\
\hline No SEN vs. SEN-E/BD & -1.82 & -2.30 & -1.35 & $<0.000$ \\
\hline \multicolumn{5}{|c|}{ Internalizing Problems (IP) } \\
\hline SEN-E/BD vs. Other SEN & 0.72 & 0.14 & 1.29 & 0.010 \\
\hline No SEN vs. Other SEN & -0.45 & -1.05 & 0.14 & 0.174 \\
\hline No SEN vs. SEN-E/BD & -1.17 & -1.60 & -0.74 & $<0.000$ \\
\hline \multicolumn{5}{|c|}{ School Related Behavior (P-SRB) } \\
\hline SEN-E/BD vs. Other SEN & -0.71 & -1.41 & -0.02 & 0.043 \\
\hline No SEN vs. Other SEN & 0.78 & 0.06 & 1.49 & 0.031 \\
\hline No SEN vs. SEN-E/BD & 1.49 & 0.97 & 2.01 & $<0.000$ \\
\hline \multicolumn{5}{|c|}{ Psychosocial Threats } \\
\hline SEN-E/BD vs. Other SEN & 0.10 & -0.31 & 0.51 & 0.818 \\
\hline No SEN vs. Other SEN & -0.26 & -0.68 & 0.17 & 0.331 \\
\hline No SEN vs. SEN-E/BD & -0.36 & -0.67 & -0.05 & 0.017 \\
\hline \multicolumn{5}{|c|}{ Coping with Distance Learning } \\
\hline SEN-E/BD vs. Other SEN & -0.21 & -0.68 & 0.27 & 0.569 \\
\hline No SEN vs. Other SEN & 0.65 & 0.16 & 1.15 & 0.006 \\
\hline No SEN vs. SEN-E/BD & 0.86 & 0.50 & 1.22 & $<0.001$ \\
\hline
\end{tabular}

\subsection{Psychosocial Threats and Coping with Distance Learning}

Students' psychosocial threats and coping with distance learning during lockdown varied across the groups (Table 4). Students with SEN-E/BD had higher psychosocial threats during lockdown than students with no SEN, and students without SEN coped significantly better with the distance learning situation than students with SEN (Table 5).

\subsection{Effect of COVID-19-Induced School Lockdowns}

As mentioned above, teachers reported a negative effect of the lockdown on P-SRB across all groups, as well as a negative effect on EP and IP in students with SEN (Table 3). To test for predictors of these effects, stepwise regression analysis was conducted for these measurements (Tables 6-8). Step 1 contained students' SEN only. SEN was dummy coded into the two dichotomous variables, SEN-E/BD and Other SEN. In step 2, psychosocial threats and coping with distance learning during lockdown were added. Step 3 additionally contained teachers' perception of student-teacher relationship work. However, step 1 resulted in a poor $R^{2}$ for all three scales (EP, IP, P-SRB). Adding the predictors in step 2 led to a significant increase in $R^{2}$, while step 3 did only for P-SRB as the criterion.

The lockdown effect on EP was best predicted by the regression model specified in step 2 (see Table 6 ), which explained $11.7 \%$ of the variance. However, the only predictor with a significant contribution here was students' psychosocial threats during lockdown $(\beta=0.21)$. This means that students who were confronted with a higher number of psychosocial threats during the lockdown were also at a higher risk of increased EP after the lockdown. Similarly, the best model for the lockdown effect on IP was step 2 (see Table 7) with $10.5 \%$ of explained variance. Students with SEN-E/BD $(\beta=0.19)$ and those who were confronted with more psychosocial threats during lockdown $(\beta=0.22)$ were at a higher risk of increased IP after lockdown.

Adding teachers' self-perceived student-teacher relationship as a predictor in step 3 led to a significant increase in explained variance for the lockdown effect on P-SRB $\left(R^{2}=0.181\right.$, see Table 8$)$. When teachers reported a stronger focus on the student-teacher relationship, the decrease in their students' P-SRB was reduced $(\beta=0.20)$. Such a protective 
effect on P-SRB was also apparent for students' coping with distance learning as a predictor $(\beta=0.27)$. However, psychosocial threats during lockdown were predictive as a risk factor for decreased P-SRB after the lockdown $(\beta=-0.24)$.

Table 6. Regression results using the lockdown effect on EP as the criterion.

\begin{tabular}{|c|c|c|c|c|c|c|c|c|c|}
\hline Predictor & $b$ & $95 \%$ CI & $\beta$ & $95 \%$ CI & $s r^{2}$ & $95 \%$ CI & $r$ & Fit & Difference \\
\hline $\begin{array}{c}\text { Step 1: } \\
\text { (Intercept) }\end{array}$ & 0.02 & {$[-0.10,0.14]$} & & & & & & & \\
\hline$S E N-E / B D=Y$ & $0.16^{*}$ & {$[0.01,0.32]$} & 0.18 & {$[0.01,0.34]$} & 0.03 & {$[0.00,0.10]$} & 0.14 & & \\
\hline \multirow[t]{2}{*}{ Other SEN = Y } & 0.12 & {$[-0.05,0.30]$} & 0.10 & {$[-0.05,0.25]$} & 0.01 & {$[0.00,0.05]$} & 0.03 & & \\
\hline & & & & & & & & $\begin{array}{l}R^{2}=0.029 \\
{[0.00,0.10]}\end{array}$ & \\
\hline
\end{tabular}

\begin{tabular}{cccccccc}
\hline Step 2: & & & & & & & \\
(Intercept) & 0.12 & {$[-0.13,0.37]$} & & & & \\
SEN-E/BD $=$ Y & 0.06 & {$[-0.10,0.22]$} & 0.07 & {$[-0.13,0.24]$} & 0.00 & {$[0.00,0.04]$} & 0.14 \\
Other SEN $=$ Y & 0.05 & {$[-0.14,0.25]$} & 0.04 & {$[-0.13,0.19]$} & 0.00 & {$[0.00,0.03]$} & 0.03 \\
CDL & -0.07 & {$[-0.14,0.00]$} & -0.15 & {$[-0.32,0.00]$} & 0.02 & {$[0.00,0.07]$} & $-0.28^{* *}$ \\
PST & $0.11^{*}$ & {$[0.02,0.20]$} & 0.21 & {$[0.04,0.37]$} & 0.03 & {$[0.00,0.10]$} & $0.30 *$
\end{tabular}

\begin{tabular}{cccccccc}
\hline $\begin{array}{c}\text { Step 3: } \\
\text { (Intercept) }\end{array}$ & 0.51 & {$[-0.10,1.09]$} & & & & & \\
SEN-E/BD $=Y$ & 0.07 & {$[-0.10,0.25]$} & 0.08 & {$[-0.13,0.27]$} & 0.00 & {$[0.00,0.05]$} & 0.14 \\
Other SEN $=Y$ & 0.04 & {$[-0.16,0.22]$} & 0.03 & {$[-0.13,0.18]$} & 0.00 & {$[0.00,0.03]$} & 0.03 \\
CDL & -0.07 & {$[-0.14,-0.00]$} & -0.16 & {$[-0.33,-0.00]$} & 0.02 & {$[0.00,0.07]$} & $-0.28^{* *}$ \\
PST & $0.11^{*}$ & {$[0.03,0.20]$} & 0.21 & {$[0.05,0.36]$} & 0.03 & {$[0.00,0.09]$} & $0.30^{* *}$ \\
$\begin{array}{c}\text { Student-teacher } \\
\text { relationship }\end{array}$ & -0.12 & {$[-0.30,0.07]$} & -0.11 & {$[-0.25,0.08]$} & 0.01 & {$[0.00,0.06]$} & -0.06
\end{tabular}

$$
\begin{array}{cc}
R^{2}=0.127^{* *} & \Delta R^{2}=0.011 \\
{[0.07,0.25]} & {[0.00,0.06]}
\end{array}
$$

Note. A significant $b$-weight indicates that the $\beta$-weight and semi-partial correlation is also significant. $b$ represents unstandardized regression weights. $\beta$ indicates the standardized regression weights. $s r^{2}$ represents the semi-partial correlation squared; $r$ represents the zero-order correlation. The amount of $95 \%$ CI represents the $95 \%$ confidence interval. Square brackets are used to enclose the lower and upper limits of a confidence interval. EP represents "Externalizing Problems", SEN represents "Special Educational Needs", E/BD represents "Emotional and Behavioral Disorders", CDL represents "Coping with Distance Learning", PST represents "Psychosocial Threats". * indicates $p<0.05,{ }^{* *}$ indicates $p<0.01$.

Table 7. Regression results using the lockdown effect on IP as the criterion.

\begin{tabular}{|c|c|c|c|c|c|c|c|c|c|}
\hline $\begin{array}{l}\text { Step 2: } \\
\text { (Intercept) }\end{array}$ & -0.13 & {$[-0.32,0.07]$} & & & & & & & \\
\hline SEN-E $/ B D=Y$ & 0.14 * & {$[0.01,0.28]$} & 0.19 & {$[0.01,0.34]$} & 0.03 & {$[0.00,0.08]$} & $0.19 *$ & & \\
\hline Other SEN $=\mathrm{Y}$ & 0.12 & {$[-0.02,0.25]$} & 0.12 & {$[-0.02,0.24]$} & 0.01 & {$[0.00,0.04]$} & 0.04 & & \\
\hline CDL & -0.00 & {$[-0.06,0.05]$} & -0.01 & {$[-0.18,0.13]$} & 0.00 & {$[0.00,0.02]$} & $-0.20 *$ & & \\
\hline PST & $0.10^{* *}$ & {$[0.04,0.17]$} & 0.22 & {$[0.10,0.35]$} & 0.04 & {$[0.01,0.09]$} & $0.27 * *$ & $\begin{array}{c}R^{2}=0.105^{* *} \\
{[0.05,0.20]}\end{array}$ & $\begin{array}{c}\Delta R^{2}=0.051^{* *} \\
{[0.01,0.14]}\end{array}$ \\
\hline
\end{tabular}

\begin{tabular}{cccccccccc}
\hline Predictor & $\boldsymbol{b}$ & $\mathbf{9 5 \%} \mathbf{C I}$ & $\boldsymbol{\beta}$ & $\mathbf{9 5 \%} \mathbf{C I}$ & $\mathbf{s r ^ { 2 }}$ & $\mathbf{9 5 \%} \mathbf{C I}$ & $\mathbf{r}$ & Fit & Difference \\
\hline Step 1: & & & & & & & & & \\
(Intercept) & -0.08 & {$[-0.19,0.01]$} & & & & & & \\
SEN-E/BD = Y & $0.18^{* *}$ & {$[0.06,0.30]$} & 0.25 & {$[0.10,0.37]$} & 0.05 & {$[0.01,0.11]$} & $0.19^{*}$ & \\
Other SEN = Y & 0.14 & {$[0.02,0.26]$} & 0.15 & {$[0.03,0.25]$} & 0.02 & {$[0.00,0.05]$} & 0.04 & $R^{2}=0.055^{* *}$ \\
& & & & & & & & & {$[0.01,0.12]$}
\end{tabular}


Table 7. Cont.

\begin{tabular}{|c|c|c|c|c|c|c|c|c|c|}
\hline Predictor & $b$ & $95 \%$ CI & $\beta$ & $95 \%$ CI & $s r^{2}$ & $95 \%$ CI & $\mathbf{r}$ & Fit & Difference \\
\hline $\begin{array}{c}\text { Step 3: } \\
\text { (Intercept) }\end{array}$ & 0.10 & {$[-0.36,0.54]$} & & & & & & & \\
\hline $\mathrm{SEN}-\mathrm{E} / \mathrm{BD}=\mathrm{Y}$ & 0.14 * & {$[-0.01,0.30]$} & 0.20 & {$[-0.02,0.37]$} & 0.03 & {$[0.00,0.09]$} & $0.19 *$ & & \\
\hline Other SEN $=\mathrm{Y}$ & 0.11 & {$[-0.03,0.23]$} & 0.11 & {$[-0.03,0.23]$} & 0.01 & {$[0.00,0.04]$} & 0.04 & & \\
\hline CDL & -0.01 & {$[-0.06,0.05]$} & -0.02 & {$[-0.19,0.13]$} & 0.00 & {$[0.00,0.03]$} & $-0.20 *$ & & \\
\hline PST & $0.10 * *$ & {$[0.03,0.17]$} & 0.22 & {$[0.09,0.36]$} & 0.04 & {$[0.01,0.10]$} & $0.27^{* *}$ & & \\
\hline \multirow{2}{*}{$\begin{array}{l}\text { Student-teacher } \\
\text { relationship }\end{array}$} & -0.07 & {$[-0.23,0.09]$} & -0.08 & {$[-0.23,0.12]$} & 0.01 & {$[0.00,0.05]$} & -0.04 & & \\
\hline & & & & & & & & $\begin{array}{c}R^{2}=0.111 \text { ** } \\
{[0.06,0.22]}\end{array}$ & $\begin{array}{c}\Delta R^{2}=0.005 \\
{[0.00,0.05]}\end{array}$ \\
\hline
\end{tabular}

Note. A significant $b$-weight indicates that the $\beta$-weight and semi-partial correlation are also significant. $b$ represents unstandardized regression weights. $\beta$ indicates the standardized regression weights. $s r^{2}$ represents the semi-partial correlation squared; $r$ represents the zero-order correlation. The amount of $95 \%$ CI represents the $95 \%$ confidence interval. Square brackets are used to enclose the lower and upper limits of a confidence interval. IP represents "Internalizing Problems", SEN represents "Special Educational Needs", E/BD represents "Emotional and Behavioral Disorders", CDL represents "Coping with Distance Learning", PST represents "Psychosocial Threats". $*$ indicates $p<0.05, * *$ indicates $p<0.01$.

Table 8. Regression results using the lockdown effect on P-SRB as the criterion.

\begin{tabular}{cccccccccc}
\hline Predictor & $\boldsymbol{b}$ & $\mathbf{9 5 \%} \mathbf{C I}$ & $\boldsymbol{\beta}$ & $\mathbf{9 5 \%} \mathbf{C I}$ & $\boldsymbol{s \boldsymbol { r } ^ { 2 }}$ & $\mathbf{9 5 \%} \mathbf{C I}$ & $\mathbf{r}$ & Fit & Difference \\
\hline $\begin{array}{c}\text { Step 1: } \\
\text { (Intercept) }\end{array}$ & -0.12 & {$[-0.25,0.01]$} & & & & & & & \\
SEN-E/BD = Y & -0.04 & {$[-0.20,0.12]$} & -0.04 & {$[-0.20,0.12]$} & 0.00 & {$[0.00,0.04]$} & -0.05 & \\
Other SEN = Y & 0.01 & {$[-0.18,0.21]$} & 0.01 & {$[-0.13,0.16]$} & 0.00 & {$[0.00,0.02]$} & 0.03 & $R^{2}=0.002$ \\
& & & & & & & & & {$[0.00,0.04]$}
\end{tabular}

\begin{tabular}{|c|c|c|c|c|c|c|c|c|c|}
\hline $\begin{array}{c}\text { Step 2: } \\
(\text { Intercept) } \\
\text { SEN-E/BD = Y } \\
\text { Other SEN = Y } \\
\text { CDL } \\
\text { PST }\end{array}$ & $\begin{array}{c}-0.34^{*} \\
0.12 \\
0.13 \\
0.13^{* *} \\
-0.15^{* *}\end{array}$ & $\begin{array}{c}{[-0.59,-0.06]} \\
{[-0.06,0.30]} \\
{[-0.07,0.34]} \\
{[0.05,0.19]} \\
{[-0.26,-0.05]}\end{array}$ & $\begin{array}{l}0.12 \\
0.10 \\
0.25 \\
-0.24\end{array}$ & $\begin{array}{c}{[-0.05,0.29]} \\
{[-0.05,0.25]} \\
{[0.09,0.39]} \\
{[-0.39,-0.08]}\end{array}$ & $\begin{array}{l}0.01 \\
0.01 \\
0.04 \\
0.04\end{array}$ & $\begin{array}{l}{[0.00,0.06]} \\
{[0.00,0.05]} \\
{[0.01,0.10]} \\
{[0.00,0.11]}\end{array}$ & $\begin{array}{c}-0.05 \\
0.03 \\
0.32^{* *} \\
-0.34^{* *}\end{array}$ & $\begin{array}{c}R^{2}=0.158^{* *} \\
{[0.08,0.28]}\end{array}$ & $\begin{array}{c}\Delta R^{2}=0.156^{* *} \\
{[0.07,0.28]}\end{array}$ \\
\hline $\begin{array}{c}\text { Step 3: } \\
\text { (Intercept) } \\
\text { SEN-E/BD = Y } \\
\text { Other SEN = Y } \\
\text { CDL } \\
\text { PST } \\
\text { Student-teacher } \\
\text { relationship }\end{array}$ & $\begin{array}{l}-1.01^{* *} \\
0.11 \\
0.16 \\
0.14^{* *} \\
-0.15^{* *} \\
0.20^{*}\end{array}$ & $\begin{array}{c}{[-1.76,-0.33]} \\
{[-0.06,0.29]} \\
{[-0.03,0.35]} \\
{[0.06,0.21]} \\
{[-0.26,-0.05]} \\
{[0.01,0.43]}\end{array}$ & $\begin{array}{l}0.11 \\
0.11 \\
0.27 \\
-0.24 \\
0.16\end{array}$ & $\begin{array}{c}{[-0.06,0.30]} \\
{[-0.03,0.25]} \\
{[0.12,0.41]} \\
{[-0.39,-0.09]} \\
{[0.01,0.30]}\end{array}$ & $\begin{array}{l}0.01 \\
0.01 \\
0.05 \\
0.04 \\
0.02\end{array}$ & $\begin{array}{l}{[0.00,0.06]} \\
{[0.00,0.05]} \\
{[0.01,0.11]} \\
{[0.01,0.11]} \\
{[0.00,0.08]}\end{array}$ & $\begin{array}{c}-0.05 \\
0.03 \\
0.32^{* *} \\
-0.34^{* *} \\
0.11\end{array}$ & $\begin{array}{c}R^{2}=0.181 * * \\
{[0.10,0.31]}\end{array}$ & $\begin{array}{c}\Delta R^{2}=0.023 * \\
{[0.00,0.08]}\end{array}$ \\
\hline
\end{tabular}

Note. A significant $b$-weight indicates that the $\beta$-weight and semi-partial correlation are also significant. $b$ represents unstandardized regression weights. $\beta$ indicates the standardized regression weights. $s r^{2}$ represents the semi-partial correlation squared; $r$ represents the zero-order correlation. The amount of $95 \%$ CI represents the $95 \%$ confidence interval. Square brackets are used to enclose the lower and upper limits of a confidence interval. P-SRB represents "Positive School Related Behavior", SEN represents "Special Educational Needs", E/BD represents "Emotional and Behavioral Disorders", CDL represents "Coping with Distance Learning", PST represents "Psychosocial Threats".* indicates $p<0.05,{ }^{* *}$ indicates $p<0.01$.

\section{Discussion}

Differences between the three groups in the current situation were to be expected, given the underlying construct of SEN-E/BD presented in prior research (e.g., Dasioti and Kolaitis 2018; DeVries et al. 2018; Schwab et al. 2016). Furthermore, our results indicate that SEN-E/BD students were confronted with more psychosocial threats during the school lockdown than other students, and had fewer individual resources to cope with distance learning. However, there was no evidence for the severe effects of the school lockdown on social and emotional variables. Moreover, the difference was marginal between SEN-E/BD students, other SEN students, and students without SEN regarding the teacher-rated effects of school lockdown. Hence, further research must focus on a wider variety of factors 
influencing the effect of school lockdown, instead of focusing exclusively on SEN-E/BD. Since the findings were partly contrary to the self-reported or parent-reported effects of the lockdown situation (Ravens-Sieberer et al. 2020, 2021; Langmeyer et al. 2020; Pearcey et al. 2020), an explanation of these discrepancies has yet to be analyzed. Therefore, several hypotheses might be possible. First, there could be general discrepancies between parent-, self-, and teacher-reports. This situation might be the case for the DBR-PUTSIE, since the SDQ parent- and self-reported scores differed slightly (Arman et al. 2013), and the SDQ teacher- and parent-reports showed only low to moderate agreement (Cheng et al. 2018; Stone et al. 2010). Second, this study analyzed the situation after the school lockdown period, while other studies (Ravens-Sieberer et al. 2020, 2021; Langmeyer et al. 2020) analyzed the situation during the lockdown. Third, Langmeyer et al. (2020) did not compare their results to the situation before the lockdown, and Ravens-Sieberer et al. (2020; 2021) based their comparison on neither retrospective data nor longitudinal data but on a comparison of their cross-sectional data with data from prior cross-sectional studies. Fourth, as these studies (Ravens-Sieberer et al. 2020, 2021; Langmeyer et al. 2020) mostly considered children and adolescents without addressing SEN, the finding that the lockdown had only mild effects for SEN students is consistent with Pearcey et al. (2020) and Chawla et al. (2021). Hence, results from several perspectives must be triangulated and completed via in-depth insights from qualitative research.

As expected, the psychosocial threats students faced in their living environment during the lockdown were the most prevalent risk factor for the negative effects of the lockdown on social and emotional problems. Moreover, having the inner resources to master the distance-learning situation was a protective factor for many aspects of social and emotional development. However, the teacher-level predictors did not have the expected effect from a theoretical perspective, and student-teacher relationships had marginal protective effects.

The finding that psychosocial threats during lockdown are a strong predictor for an impact on the social-emotional problems of children and adolescents is consistent with prior findings and research-based assumptions, as mentioned in the introduction (Clemens et al. 2020; Fontanesi et al. 2020; Mechili et al. 2021; Spinelli et al. 2020). However, according to prior research (Lee 2012; Pianta and Stuhlman 2004; Wanders et al. 2020), the student-teacher relationship scale had a surprisingly small impact. This situation can be explained in two ways. First, psychosocial threats and other student-level predictors might have such an impact that teachers' student-teacher relationships cannot compensate for them, especially when a lockdown makes the situation more complicated. Second, the student-teacher relationship scale seeks teachers' perspectives (students' perspectives might be different).

\subsection{Limitations}

Several methodical limitations must be considered. First, our sample size was small, with 94 teachers. Furthermore, it was an ad-hoc sample recruited via social media, the local press, and mailing lists. Several factors of teacher variables may confound the willingness to participate in this online survey and their perception of students' EB/D. Hence, the influence of a selection bias must be considered, which limits the external validity of our findings when addressing students in general. Second, as discussed, the findings employed teachers' perspectives, which may under- or overestimate emotional problems relative to self- or parent-reports (Arman et al. 2013; Cheng et al. 2018; Stone et al. 2010; Langmeyer et al. 2020). This also indicates a further limitation that we could not sufficiently collect data on students' socioeconomic situation and their families' situations. Third, to evaluate changes in the dependent variables, we used retrospective ratings. Thus, we have no longitudinal data and must consider that a recall bias may influence teachers' retrospective perceptions. Finally, the adequate psychometric quality of the DBR-PUTSIE was assumed based on findings from similar instruments but was not evaluated for this measurement; 
however, the PUTSIE-scales demonstrated good reliability in our study (see Appendix B Tables A1 and A2).

Although we were interested in comparing the period before and after school lockdown, we could not distinguish between the effects of the school lockdown and the pandemic. It is reasonable to assume that IP would be affected by several factors related to the pandemic (e.g., the fear of getting infected or losing beloved family members to a fatal COVID-19 infection). Thus, we cannot posit any causal effect of the school lockdown but only the coincidence.

\subsection{Conclusions}

This study found evidence for marginal effects of the school lockdown on students $\mathrm{E} / \mathrm{BD}$ rather than for severe issues. However, our findings suggest that the psychosocial situation of children and adolescents during the pandemic is a potential risk factor that should be monitored by teachers, school social workers, and school psychologists. Moreover, measures should be taken to strengthen student-teacher relationships even over a distance. Hence, where necessary, opportunities for personal accompaniment and support for students, and even parents, must be provided.

As indicated above, further research is required that triangulates the perspectives of teachers, students, and parents. Additionally, it would be helpful to implement a longitudinal design to evaluate the continuing effects of repeated lockdowns. Further, we gathered qualitative data providing in-depth insights into the teachers' perspectives in a follow-up inquiry via qualitative interviews. The interviews are currently under preparation for analysis.

Author Contributions: All authors D.S., D.L. contributed equally to the research project and the publication. All authors have read and agreed to the published version of the manuscript.

Funding: This study was funded by Paderborn University. Publication costs were funded by Deutsche Forschungsgemeinschaft (DFG), grant No. 453372524.

Data Availability Statement: The datasets generated for this study, the full questionnaire, and the R input code can be found in the OSF repository: https:/ / osf.io/T3P6A/ (accessed 18 October 2021).

Acknowledgments: An extended prior version of this paper has been published as a preprint on EdArXiv (Scheer and Laubenstein 2021).

Conflicts of Interest: The authors declare that the research was conducted in the absence of any commercial or financial relationships that could be construed as a potential conflict of interest.

\section{Appendix A. Instructions for the Survey Scenarios}

Appendix A.1. Instructions for Scenario 1

\section{Prepare class list for anonymous student selection.}

1. Please have a copy or a printout of your class list in hand. You should have the following information ready during the survey:

- Age and gender of the students;

- Grade level;

- Existence of an official diagnosis of special educational needs (with all types of SEN assigned to the student);

- Presence of one or more of the following diagnoses: autism spectrum disorder, $\mathrm{ADD}, \mathrm{ADHD}$, auditory processing and perception disorder, learning disabilities.

2. Please number all students on your class list who have no official diagnosis of special educational needs in emotional and behavioral disorders. Start with ' 1 '.

\section{Selection of students to be assessed.}

1. On the next page you will see which students from your class list you should anonymously assess during the survey. 
2. Please mark these students on your list so that you can find them more quickly afterwards.

Appendix A.2. Instructions for Scenario 2

Prepare class list for anonymous student selection.

Please have a copy or a printout of your class list in hand. You should have the following information ready during the survey:

- $\quad$ Age and gender of the students;

- Grade level;

- Existence of an official diagnosis of special educational needs (with all types of SEN assigned to the student);

- Presence of one or more of the following diagnoses: autism spectrum disorder, ADD, ADHD, auditory processing and perception disorder, learning disabilities.

\section{Selection of students to be assessed.}

1. On the next page you will see which students from your class list you should anonymously assess during the survey.

2. Please mark these students on your list so that you can find them more quickly afterwards.

\section{Appendix A.3. Instructions for Scenario 3}

\section{Prepare class list for anonymous student selection.}

1. Please have a copy or a printout of your class list in hand. You should have the following information ready during the survey:

- Age and gender of the students;

- Grade level;

- Existence of an official diagnosis of special educational needs (with all types of SEN assigned to the student);

- Presence of one or more of the following diagnoses: autism spectrum disorder, ADD, ADHD, auditory processing and perception disorder, learning disabilities.

2. Please number all students on your class list who have an official diagnosis of special educational needs in emotional and behavioral disorders. Start with ' 1 '.

3. Please strike out all students on your class list who have no official diagnosis of special educational needs in emotional and behavioral disorders. Start with ' 1 '.

Selection of students to be assessed.

1. On the next page you will see which students from your class list you should anonymously assess during the survey.

2. Please mark these students on your list so that you can find them more quickly afterwards.

Appendix A.4. Instructions for Scenario 4

\section{Prepare class list for anonymous student selection.}

1. Please have a copy or a printout of your class list in hand. You should have the following information ready during the survey:

- Age and gender of the students;

- Grade level;

- Existence of an official diagnosis of special educational needs (with all types of SEN assigned to the student);

- Presence of one or more of the following diagnoses: autism spectrum disorder, ADD, ADHD, auditory processing and perception disorder, learning disabilities.

2. Please number your entire class list. Start with ' 1 '. 


\section{Selection of students to be assessed.}

1. On the next page you will see which students from your class list you should anonymously assess during the survey.

2. Please mark these students on your list so that you can find them more quickly afterwards.

\section{Appendix B. Measurements}

Table A1. Item and scale properties of the measurements for the current situation of EP, IP, and P-SRB.

\begin{tabular}{|c|c|c|c|c|c|c|c|}
\hline Item & $\mathbf{N}$ & $\mathbf{M}$ & SD & Min & Max & SE & $r_{\text {it }}$ \\
\hline \multicolumn{8}{|c|}{ Positive School Related Behavior (P-SRB: $\alpha=0.88$ ) } \\
\hline Raises hand before speaking in class & 173 & 3.12 & 1.79 & 0 & 6 & 0.1362 & 0.630 \\
\hline Follows rules for speaking in class (i.e., raises hand) & 173 & 3.36 & 1.82 & 0 & 6 & 0.1387 & 0.775 \\
\hline Concentrates on schoolwork & 173 & 3.04 & 1.76 & 0 & 6 & 0.1341 & 0.905 \\
\hline Works quietly at desk & 173 & 3.40 & 1.85 & 0 & 6 & 0.1410 & 0.819 \\
\hline Participates in classes & 173 & 3.30 & 1.79 & 0 & 6 & 0.1360 & 0.860 \\
\hline \multicolumn{8}{|c|}{ Externalizing Problems (EP: $\alpha=0.95)$} \\
\hline Is easily annoyed & 173 & 2.94 & 2.08 & 0 & 6 & 0.1581 & 0.733 \\
\hline Refuses to obey rules & 172 & 2.03 & 1.80 & 0 & 6 & 0.1373 & 0.839 \\
\hline Resists instructions from authority figures & 173 & 1.91 & 1.87 & 0 & 6 & 0.1425 & 0.821 \\
\hline Annoys others on purpose & 172 & 2.26 & 1.92 & 0 & 6 & 0.1462 & 0.664 \\
\hline Makes careless mistakes in schoolwork & 172 & 3.31 & 1.73 & 0 & 6 & 0.1318 & 0.584 \\
\hline Has trouble maintaining level of concentration while performing tasks & 173 & 3.32 & 1.96 & 0 & 6 & 0.1489 & 0.786 \\
\hline Does not seem to listen when being addressed by others & 173 & 2.23 & 1.85 & 0 & 6 & 0.1404 & 0.718 \\
\hline Frequently quits tasks early & 173 & 2.98 & 1.93 & 0 & 6 & 0.1470 & 0.640 \\
\hline Has difficulty getting organized & 172 & 3.15 & 2.13 & 0 & 6 & 0.1624 & 0.751 \\
\hline Easily distracted & 172 & 3.52 & 2.01 & 0 & 6 & 0.1533 & 0.845 \\
\hline Fidgets with hands and feet & 173 & 2.05 & 2.06 & 0 & 6 & 0.1567 & 0.803 \\
\hline Stands up in situations where sitting is expected & 173 & 1.65 & 1.91 & 0 & 6 & 0.1450 & 0.823 \\
\hline Acts as if they are "driven" & 172 & 1.60 & 1.93 & 0 & 6 & 0.1470 & 0.758 \\
\hline Talks a lot & 172 & 2.59 & 1.88 & 0 & 6 & 0.1433 & 0.568 \\
\hline Has difficulty waiting for their turn & 173 & 2.23 & 1.98 & 0 & 6 & 0.1507 & 0.770 \\
\hline \multicolumn{8}{|c|}{ Internalizing Problems (IP: $\alpha=0.85$ ) } \\
\hline Often seems sad & 172 & 1.94 & 1.84 & 0 & 6 & 0.1400 & 0.720 \\
\hline Shows decreased interest in activities & 173 & 2.08 & 1.84 & 0 & 6 & 0.1401 & 0.775 \\
\hline Has trouble making decisions & 173 & 2.23 & 1.73 & 0 & 6 & 0.1315 & 0.673 \\
\hline Is afraid of social situations & 172 & 2.06 & 1.94 & 0 & 6 & 0.1477 & 0.729 \\
\hline Complains of physical discomfort & 173 & 1.45 & 1.75 & 0 & 6 & 0.1328 & 0.597 \\
\hline Works mostly alone, prefers to be alone & 173 & 2.58 & 1.70 & 0 & 6 & 0.1291 & 0.579 \\
\hline Plays mostly alone, prefers to be alone & 173 & 1.75 & 1.54 & 0 & 6 & 0.1169 & 0.584 \\
\hline Teased or bullied by classmates & 173 & 1.47 & 1.52 & 0 & 6 & 0.1152 & 0.610 \\
\hline
\end{tabular}

Note. $r_{\text {it }}$ represents the corrected item-total correlation. $\alpha$ represents Cronbach's $\alpha$ as a measure of reliability. 
Table A2. Item and scale properties of the measurements for the lockdown effect on EP, IP, and P-SRB.

\begin{tabular}{|c|c|c|c|c|c|c|c|}
\hline Item & $\mathbf{N}$ & $\mathbf{M}$ & SD & Min & Max & SE & $r_{\text {it }}$ \\
\hline \multicolumn{8}{|c|}{ Positive School Related Behavior (P-SRB: $\alpha=0.82$ ) } \\
\hline Raises hand before speaking in class & 173 & -0.10 & 0.63 & -2 & 2 & 0.0476 & 0.530 \\
\hline Follows rules for speaking in class (i.e., raises hand) & 173 & -0.09 & 0.58 & -2 & 1 & 0.0440 & 0.620 \\
\hline Concentrates on schoolwork & 173 & -0.23 & 0.75 & -2 & 2 & 0.0569 & 0.846 \\
\hline Works quietly at desk & 173 & -0.14 & 0.64 & -2 & 2 & 0.0490 & 0.734 \\
\hline Participates in classes & 173 & -0.12 & 0.71 & -2 & 2 & 0.0537 & 0.782 \\
\hline \multicolumn{8}{|c|}{ Externalizing Problems (EP: $\alpha=0.92)$} \\
\hline Is easily annoyed & 173 & 0.14 & 0.71 & -2 & 2 & 0.0542 & 0.693 \\
\hline Refuses to obey rules & 173 & 0.12 & 0.71 & -2 & 2 & 0.0537 & 0.792 \\
\hline Resists instructions from authority figures & 173 & 0.09 & 0.71 & -2 & 2 & 0.0543 & 0.840 \\
\hline Annoys others on purpose & 173 & 0.07 & 0.70 & -2 & 2 & 0.0529 & 0.638 \\
\hline Makes careless mistakes in schoolwork & 173 & 0.12 & 0.48 & -1 & 2 & 0.0365 & 0.472 \\
\hline Has trouble maintaining level of concentration while performing tasks & 173 & 0.19 & 0.68 & -2 & 2 & 0.0514 & 0.673 \\
\hline Does not seem to listen when being addressed by others & 173 & 0.09 & 0.50 & -2 & 1 & 0.0383 & 0.688 \\
\hline Frequently quits tasks early & 173 & 0.18 & 0.67 & -2 & 2 & 0.0512 & 0.673 \\
\hline Has difficulty getting organized & 173 & 0.21 & 0.70 & -2 & 2 & 0.0533 & 0.740 \\
\hline Easily distracted & 173 & 0.28 & 0.70 & -2 & 2 & 0.0533 & 0.786 \\
\hline Fidgets with hands and feet & 173 & 0.06 & 0.55 & -2 & 2 & 0.0416 & 0.672 \\
\hline Stands up in situations where sitting is expected & 173 & 0.06 & 0.57 & -2 & 2 & 0.0432 & 0.740 \\
\hline Acts as if they are "driven" & 173 & 0.02 & 0.56 & -2 & 2 & 0.0426 & 0.711 \\
\hline Talks a lot & 172 & 0.08 & 0.59 & -1 & 2 & 0.0452 & 0.357 \\
\hline Has difficulty waiting for their turn & 173 & 0.04 & 0.50 & -2 & 2 & 0.0379 & 0.609 \\
\hline \multicolumn{8}{|c|}{ Internalizing Problems (IP: $\alpha=0.84)$} \\
\hline Often seems sad & 173 & 0.13 & 0.62 & -2 & 2 & 0.0471 & 0.687 \\
\hline Shows decreased interest in activities & 173 & 0.13 & 0.55 & -2 & 2 & 0.0418 & 0.699 \\
\hline Has trouble making decisions & 173 & 0.01 & 0.42 & -2 & 2 & 0.0317 & 0.599 \\
\hline Is afraid of social situations & 173 & 0.06 & 0.49 & -2 & 2 & 0.0373 & 0.764 \\
\hline Complains of physical discomfort & 173 & 0.04 & 0.50 & -2 & 2 & 0.0379 & 0.564 \\
\hline Works mostly alone, prefers to be alone & 173 & 0.00 & 0.52 & -2 & 2 & 0.0393 & 0.522 \\
\hline Plays mostly alone, prefers to be alone & 173 & -0.09 & 0.56 & -2 & 2 & 0.0428 & 0.639 \\
\hline Teased or bullied by classmates & 173 & -0.10 & 0.49 & -2 & 1 & 0.0373 & 0.648 \\
\hline
\end{tabular}

Table A3. Item and scale properties of the measurements for students' coping with distance learning and psychosocial threats during lockdown.

\begin{tabular}{|c|c|c|c|c|c|c|c|}
\hline Item & $\mathbf{N}$ & $\mathbf{M}$ & SD & Min & Max & SE & $r_{\text {it }}$ \\
\hline \multicolumn{8}{|c|}{ Coping with Distance Learning $(\alpha=0.83)$} \\
\hline (-) The student seemed to believe they were on vacation. & 173 & 2.07 & 1.34 & 0 & 4 & 0.1019 & -0.726 \\
\hline (-) The student felt very stressed in the situation. & 171 & 1.95 & 1.15 & 0 & 4 & 0.0881 & -0.256 \\
\hline $\begin{array}{l}\text { The student had enough opportunities to work on the } \\
\text { computer/laptop/tablet at home. }\end{array}$ & 172 & 2.39 & 1.49 & 0 & 4 & 0.1135 & 0.551 \\
\hline $\begin{array}{l}\text { The student was looking forward to the other learning styles/learning } \\
\text { methods (e.g., e-learning). }\end{array}$ & 173 & 1.57 & 1.26 & 0 & 4 & 0.0960 & 0.836 \\
\hline The student actively worked on the tasks at home. & 173 & 1.98 & 1.41 & 0 & 4 & 0.1072 & 0.740 \\
\hline $\begin{array}{l}\text { The student managed to get involved in the other learning } \\
\text { styles/learning methods. }\end{array}$ & 173 & 1.65 & 1.37 & 0 & 4 & 0.1042 & 0.951 \\
\hline \multicolumn{8}{|c|}{ Psychosocial Threats $(\alpha=0.81)$} \\
\hline $\begin{array}{c}\text { The student is at risk of psychological or physical violence in the } \\
\text { out-of-school living environment. }\end{array}$ & 172 & 0.69 & 0.96 & 0 & 4 & 0.0731 & 0.859 \\
\hline The student witnesses violence in the out-of-school environment. & 171 & 0.61 & 0.94 & 0 & 3 & 0.0715 & 0.789 \\
\hline (-) The student's basic needs are ensured during the school closure. & 173 & 3.49 & 0.91 & 0 & 4 & 0.0689 & -0.365 \\
\hline $\begin{array}{c}\text { The student is at risk of emotional neglect in the out-of-school } \\
\text { environment. }\end{array}$ & 172 & 0.94 & 1.17 & 0 & 4 & 0.0889 & 0.838 \\
\hline The student is threatened with social isolation outside of school. & 172 & 1.11 & 1.27 & 0 & 4 & 0.0970 & 0.618 \\
\hline
\end{tabular}

Note. $r_{\text {it }}$ represents the corrected item-total correlation. $\alpha$ represents Cronbach's $\alpha$ as a measure of reliability. (-) indicates that the item was recoded for building the overall scale. 
Table A4. Item and scale properties of the measurements for teachers' perception of student-teacher relationship $(\alpha=0.78)$.

\begin{tabular}{cccccccc}
\hline Item & $\mathbf{N}$ & $\mathbf{M}$ & $\mathbf{S D}$ & Min & Max & SE & $\boldsymbol{r}_{\text {it }}$ \\
\hline Item_01 & 173 & 3.41 & 0.59 & 2 & 4 & 0.0449 & 0.298 \\
Item_02 & 173 & 3.40 & 0.60 & 2 & 4 & 0.0455 & 0.545 \\
Item_03 & 173 & 3.10 & 0.77 & 1 & 4 & 0.0586 & 0.403 \\
Item_04 & 173 & 3.51 & 0.60 & 2 & 4 & 0.0454 & 0.670 \\
Item_05 & 173 & 2.98 & 0.74 & 1 & 4 & 0.0559 & 0.596 \\
Item_06 & 170 & 2.56 & 0.73 & 1 & 4 & 0.0559 & 0.779 \\
Item_07 & 173 & 3.46 & 0.52 & 2 & 4 & 0.0397 & 0.454 \\
Item_08 & 173 & 3.38 & 0.64 & 1 & 4 & 0.0487 & 0.541 \\
Item_09 & 173 & 3.39 & 0.59 & 2 & 4 & 0.0445 & 0.657 \\
\hline Note. $r_{\text {it }}$ represents the corrected item-total correlation. $\alpha$ represents Cronbach's $\alpha$ as a measure of reliability.
\end{tabular}

\section{References}

Arman, Soroor, Afsaneh Karbasi Amel, and Mohamad Reza Maracy. 2013. Comparison of Parent Adolescent Scores on Strengths and Difficulties Questionnaire. Journal of Research in Medical Sciences 18: 501-5.

Baginsky, Mary, and Jill Manthorpe. 2021. The Impact of Covid-19 on Children's Social Care in England. Child Abuse E Neglect 116: 104739. [CrossRef]

Baumann, Menno. 2020. Welche Pädagogisch-Psychologischen Auswirkungen Hat Quarantäne auf Kinder und Familien? [What Are the Educational-Psychological Effects of Quarantine on Children and Families?]. Publikum, November 2. Available online: https: //publikum.net/welche-padagogisch-psychologischen-auswirkungen-hat-quarantane-auf-kinder-und-familien/ (accessed on 20 September 2021).

Baumgarten, Franz, Karin Klipker, Kristin Göbel, Silke Janitza, and Heike Hölling. 2018. Der Verlauf psychischer Auffälligkeiten bei Kindern und Jugendlichen-Ergebnisse der KiGGS-Kohorte [The progress of mental disorders in children and adolescents-results of the KiGGS cohort]. Journal of Health Monitoring 3: 60-65. [CrossRef]

Blumenthal, Yvonne, Bodo Hartke, Thomas Hennemann, Clemens Hillenbrand, Gino Casale, and Marie-Christine Vierbuchen. 2020. Kinder mit Verhaltensauffälligkeiten und Emotional-Sozialen Entwicklungsstörungen: Förderung in inklusiven Schulklassen [Children with Deviant Behavior and Disorders of Emotional-Social Development: Support in Inclusive Regular Classrooms]. Stuttgart: Kohlhammer.

Börnert-Ringleb, Moritz, Gino Casale, and Clemens Hillenbrand. 2021. What Predicts Teachers' Use of Digital Learning in Germany? Examining the Obstacles and Conditions of Digital Learning in Special Education. European Journal of Special Needs Education 36: 80-97. [CrossRef]

Brooks, Samantha K., Rebecca K. Webster, Louise E. Smith, Lisa Woodland, Simon Wessely, Neil Greenberg, and Gideon James Rubin. 2020. The Psychological Impact of Quarantine and How to Reduce It: Rapid Review of the Evidence. The Lancet 395: 912-20. [CrossRef]

Casale, Gino, Moritz Börnert-Ringleb, and Clemens Hillenbrand. 2020a. Fördern auf Distanz? Sonderpädagogische Unterstützung im Lernen und in der emotional-sozialen Entwicklung während der Schulschließungen 2020 gemäß der Regelungen der Bundesländer [Support at a distance? Special educational support in learning and emotional-social development during the school closures 2020 according to the regulations of the federal states]. Zeitschrift für Heilpädagogik 71: 254-67.

Casale, Gino, Moritz Börnert-Ringleb, and Clemens Hillenbrand. 2020b. SOLVE-Sonderpädagogischer Online-Unterricht für Schüler*innen mit Lern-und Verhaltensproblemen [SOLVE-Special Education Online Lessons for Pupils with Learning and Behavioural Problems.]. Available online: https://www.researchgate.net/project/SOLVE-Sonderpaedagogischer-OnlineUnterricht-fuer-Schuelerinnen-mit-Lern-und-Verhaltensproblemen (accessed on 2 January 2021).

Chawla, Nishtha, Pawan Sharma, and Rajesh Sagar. 2021. Psychological Impact of COVID-19 on Children and Adolescents: Is There a Silver Lining? Indian Journal of Pediatrics 88: 91. [CrossRef]

Chen, I.-Hua, Chao-Ying Chen, Amir H. Pakpour, Mark D. Griffiths, and Chung-Ying Lin. 2020. Internet-Related Behaviors and Psychological Distress among Schoolchildren during COVID-19 School Suspension. Journal of the American Academy of Child and Adolescent Psychiatry 59: 1099-102. [CrossRef] [PubMed]

Cheng, Sabrina, Katherine M. Keyes, Adina Bitfoi, Mauro Giovanni Carta, Ceren Koç, Dietmar Goelitz, Roy Otten, Sigita Lesinskiene, Zlatka Mihova, Ondine Pez, and et al. 2018. Understanding Parent-Teacher Agreement of the Strengths and Difficulties Questionnaire (SDQ): Comparison across Seven European Countries. International Journal of Methods in Psychiatric Research 27: e1589. [CrossRef] [PubMed]

Christ, Theodore J., T. Chris Riley-Tillman, and Sandra M. Chafouleas. 2009. Foundation for the Development and Use of Direct Behavior Rating (DBR) to Assess and Evaluate Student Behavior. Assessment for Effective Intervention 34: 201-13. [CrossRef]

Clemens, Vera, Peter Deschamps, Jörg M. Fegert, Dimitris Anagnostopoulos, Sue Bailey, Maeve Doyle, Stephan Eliez, Anna Sofie Hansen, Johannes Hebebrand, Manon Hillegers, and et al. 2020. Potential Effects of "Social" Distancing Measures and School Lockdown on Child and Adolescent Mental Health. European Child \& Adolescent Psychiatry 29: 739-42. [CrossRef] 
Dasioti, A, and G. Kolaitis. 2018. Bullying and the Mental Health of Schoolchildren with Special Educational Needs in Primary Education. Psychiatrike = Psychiatriki 29: 149-59. [CrossRef] [PubMed]

DeVries, Jeffrey M., Stefan Voß, and Markus Gebhardt. 2018. Do Learners with Special Education Needs Really Feel Included? Evidence from the Perception of Inclusion Questionnaire and Strengths and Difficulties Questionnaire. Research in Developmental Disabilities 83: 28-36. [CrossRef] [PubMed]

Fontanesi, Lilybeth, Daniela Marchetti, Cristina Mazza, Serena Di Giandomenico, Paolo Roma, and Maria Cristina Verrocchio. 2020. The Effect of the COVID-19 Lockdown on Parents: A Call to Adopt Urgent Measures. Psychological Trauma: Theory, Research, Practice and Policy 12: S79-S81. [CrossRef] [PubMed]

Forness, Steven R., and Kenneth A. Kavale. 2000. Emotional or Behavioral Disorders: Background and Current Status of the E/BD Terminology and Definition. Behavioral Disorders 25: 264-69.

Gebhardt, Markus, Jeffrey M. DeVries, Jana Jungjohann, and Gino Casale. 2018. Questionnaire Monitoring Behavior in Schools (QMBS). Description of the Scale "Questionnaire Monitoring Behavior in Schools" (QMBS) in English and German Language. Available online: https:/ / www.researchgate.net/publication/328497065_Questionnaire_Monitoring_Behavior_in_Schools_QMBS_DBRMIS_Description_of_the_scale_Questionnaire_Monitoring_Behavior_in_Schools_QMBS_in_English_and_German_language (accessed on 21 September 2021). [CrossRef]

Gebhardt, Markus, Jeffrey M. DeVries, Jana Jungjohann, Gino Casale, Andreas Gegenfurtner, and Jörg-Tobias Kuhn. 2019. Measurement Invariance of a Direct Behavior Rating Multi Item Scale across Occasions. Social Sciences 8: 46. [CrossRef]

Goodman, Robert. 1999. The Extended Version of the Strengths and Difficulties Questionnaire as a Guide to Child Psychiatric Caseness and Consequent Burden. Journal of Child Psychology and Psychiatry and Allied Disciplines 40: 791-99. [CrossRef]

Goodman, Robert. 2005. Fragebogen zu Stärken und Schwächen (SDQ-Deu). Available online: http://sdqinfo.org/py/sdqinfo/b3.py? language $=$ German (accessed on 2 January 2021).

Graber, Kelsey, Elizabeth Mary Byrne, Emily J. Goodacre, Natalie Kirby, Krishna Kulkarni, Christine O'Farrelly, and Paul G. Ramchandani. 2020. A Rapid Review of the Impact of Quarantine and Restricted Environments on Children's Play and Health Outcomes. PsyArXiv. [CrossRef]

Henssler, Jonathan, Friederike Stock, Joris van Bohemen, Henrik Walter, Andreas Heinz, and Lasse Brandt. 2020. Mental Health Effects of Infection Containment Strategies: Quarantine and Isolation-A Systematic Review and Meta-Analysis. European Archives of Psychiatry and Clinical Neuroscience 271: 223-34. [CrossRef]

Hossain, Md Mahbub, Abida Sultana, and Neetu Purohit. 2020. Mental Health Outcomes of Quarantine and Isolation for Infection Prevention: A Systematic Umbrella Review of the Global Evidence. Epidemiology and Health 42: e2020038. [CrossRef]

Huber, Stephan Gerhard, Paula Sophie Günther, Nadine Schneider, Christoph Helm, Marius Schwander, Julia Schneider, and Jane Pruitt. 2020. COVID-19-Aktuelle Herausforderungen in Schule und Bildung [COVID-19 and current Challenges in Schools and Education]. Münster: Waxmann.

Idoiaga Mondragon, Nahia, Naiara Berasategi Sancho, Maria Dosil Santamaria, and Amaia Eiguren Munitis. 2021. Struggling to Breathe: A Qualitative Study of Children's Wellbeing during Lockdown in Spain. Psychology E Health 36: 179-94. [CrossRef]

Imran, Nazish, Irum Aamer, Muhammad Imran Sharif, Zubair Hassan Bodla, and Sadiq Naveed. 2020. Psychological Burden of Quarantine in Children and Adolescents: A Rapid Systematic Review and Proposed Solutions. Pakistan Journal of Medical Sciences 36: 1106-16. [CrossRef] [PubMed]

Jentsch, Birgit, and Brigitte Schnock. 2020. Child Welfare in the Midst of the Coronavirus Pandemic-Emerging Evidence from Germany. Child Abuse \& Neglect 110: 104716. [CrossRef]

Kultusministerkonferenz. 2019. The Education System in the Federal Republic of Germany 2016/2017. Available online: https:/ /www. kmk.org/fileadmin/Dateien/pdf/Eurydice/Bildungswesen-engl-pdfs/dossier_en_ebook.pdf (accessed on 2 January 2021).

Langmeyer, Alexandra, Angelika Guglhör-Rudan, Thorsten Naab, Marc Urlen, and Ursula Winklhofer. 2020. Kindsein in Zeiten von Corona: Erste Ergebnisse zum veränderten Alltag und zum Wohlbefinden von Kindern [Being a Child in Times of Corona: First Results on the Changed Everyday Life and Well-Being of Children.]. München: Deutsches Jugendinstitut, Available online: https: //www.dji.de/fileadmin/user_upload/dasdji/news/2020/DJI_Kindsein_Corona_Ergebnisbericht_2020.pdf (accessed on 18 October 2021).

Lee, Jung-Sook. 2012. The Effects of the Teacher-Student Relationship and Academic Press on Student Engagement and Academic performance. International Journal of Educational Research 53: 330-40. [CrossRef]

Lee, Joyce. 2020. Mental Health Effects of School Closures during Covid-19. The Lancet. Child E Adolescent Health 4: 421. [CrossRef]

LimeSurvey. 2019. Available online: https:/ / www.limesurvey.org/de/ (accessed on 20 September 2021).

Mechili, Enkeleint A., Aurela Saliaj, Fatjona Kamberi, Charis Girvalaki, Ela Peto, Athina E. Patelarou, Jorgjia Bucaj, and Evridiki Patelarou. 2021. Is the Mental Health of Young Students and Their Family Members Affected During the Quarantine Period? Evidence from the COVID-19 Pandemic in Albania. Journal of Psychiatric and Mental Health Nursing 28: 317-25. [CrossRef]

Pearcey, Samantha, Adrienne Shum, Polly Waite, Praveetha Patalay, and Cathy Creswell. 2020. Changes in Children and Young People's Emotional and Behavioural Difficulties through Lockdown. CoSPACE Report 04. Available online: https:/ / emergingminds.org. uk/wp-content/uploads/2020/06/CoSPACE-Report-4-June-2020.pdf (accessed on 21 December 2020).

Pianta, Robert C., and Megan W. Stuhlman. 2004. Teacher-Child Relationships and Children's Success in the First Years of School. School Psychology Review 33: 444-58. [CrossRef]

Pianta, Robert C., Karen M. La Paro, and Bridget K. Hamre. 2008. Classroom Assessment Scoring System: (CLASS). Baltimore: Brookes. 
R Core Team. 2020. R: A Language and Environment for Statistical Computing. Available online: https://www.r-project.org (accessed on 18 October 2021).

Ravens-Sieberer, Ulrike, Anne Kaman, Christiane Otto, Adekunle Adedeji, Janine Devine, Michael Erhart, Ann-Kathrin Napp, Marcia Becker, Ulrike Blanck-Stellmacher, Constanze Löffler, and et al. 2020. Mental Health and Quality of Life in Children and Adolescents during the COVID-19 Pandemic-Results of the COPSY Study. Deutsches Aerzteblatt International 117: 828-29. [CrossRef] [PubMed]

Ravens-Sieberer, Ulrike, Anne Kaman, Michael Erhart, Janine Devine, Robert Schlack, and Christiane Otto. 2021. Impact of the COVID-19 Pandemic on Quality of Life and Mental Health in Children and Adolescents in Germany. European Child E Adolescent Psychiatry 25: 1-11. [CrossRef]

Revelle, William. 2020. Psych: Procedures for Psychological, Psychometric, and Personality Research. Evanston: Illinois.

Röhr, Susanne, Felix Müller, Franziska Jung, Christian Apfelbacher, Andreas Seidler, and Steffi G. Riedel-Heller. 2020. Psychosoziale Folgen von Quarantänemaßnahmen bei schwerwiegenden c Coronavirus-Ausbrüchen: Ein rapid review [Psychosocial Impact of Quarantine Measures during Serious Coronavirus Outbreaks: A Rapid Review]. Psychiatrische Praxis 47: 179-89. [CrossRef]

Ron, Adrián García, and Isabel Cuéllar-Flores. 2020. Psychological Impact of Lockdown (Confinement) on Young Children and How to Mitigate its Effects: Rapid Review of the Evidence. Anales de Pediatría (English Edition) 93: 57-58. [CrossRef]

RStudio. 2020. Available online: https:/ / www.rstudio.com/ (accessed on 20 September 2021).

Scheer, David, and Désirée Laubenstein. 2021. The Impact of COVID-19 on Mental Health and Psycho-social Conditions of Students with and withound Special Educational Needs in Emotional and Behavioral Disorders in Germany. EdArXiv. [CrossRef]

Scheer, David, and Conny Melzer. 2020. Trendanalyse der KMK-Statistiken zur sonderpädagogischen Förderung 1994 bis 2019 [Trend analysis of the KMK-statistics on special needs education 1994 to 2019]. Zeitschrift für Heilpädagogik 71: 575-591. Available online: https:/ / osf.io/kzh7v/ (accessed on 20 September 2021).

Schurig, Michael, Silas Garthe-Krause, and Markus Gebhardt. 2020. Verlaufsdiagnostik für Verhalten in der Schule (dbr-putsie) [Progress diagnostics for behaviour at school (dbr-putsie)]. Available online: https://www.researchgate.net/publication/339107 254_Verlaufsdiagnostik_fur_Verhalten_in_der_Schule_DBR-PUTSIE (accessed on 20 September 2021).

Schwab, Susanne, Markus Gebhardt, Marco G. P. Hessels, and Lena Nusser. 2016. Predicting a High Rate of Self-Assessed and Parent-Assessed Peer Problems-Is It Typical for Students with Disabilities? Research in Developmental Disabilities 49-50: 196-204. [CrossRef] [PubMed]

Sharan, Pratap, and Pallavi Rajhans. 2020. Social and Psychological Consequences of "Quarantine": A Systematic Review and Application to India. Indian Journal of Social Psychiatry 36: 112-19. [CrossRef]

Sharma, Vinita, Miguel Reina Ortiz, and Nandita Sharma. 2020. Risk and Protective Factors for Adolescent and Young Adult Mental Health within the Context of COVID-19: A Perspective from Nepal. The Journal of Adolescent Health 67: 135-37. [CrossRef] [PubMed]

Singh, Shweta, Deblina Roy, Krittika Sinha, Sheeba Parveen, Ginni Sharma, and Gunjan Joshi. 2020. Impact of COVID-19 and Lockdown on Mental Health of Children and Adolescents: A Narrative Review with Recommendations. Psychiatry Research 293: 113429. [CrossRef]

Spinelli, Maria, Francesca Lionetti, Massimiliano Pastore, and Mirco Fasolo. 2020. Parents' Stress and Children's Psychological Problems in Families Facing the COVID-19 O Outbreak in Italy. Frontiers in Psychology 11: 1713. [CrossRef]

Stanley, David. 2018. ApaTables: Create American Psychological Association (APA) Style Tables. Available online: https://dstanley4 .github.io/apaTables/reference/apaTables.html (accessed on 13 October 2021).

Stone, Lisanne L., Roy Otten, Rutger C. M. E. Engels, Ad A. Vermulst, and Jan M. A. M. Janssens. 2010. Psychometric Properties of the Parent and Teacher Versions of the Strengths and Difficulties Questionnaire for 4- to 12-Year-Olds: A Review. Clinical Child and Family Psychology Review 13: 254-74. [CrossRef]

Wacker, Albrecht, Valentin Unger, and Thomas Rey. 2020. "Sind doch Corona-Ferien, oder nicht?" [It's Corona holidays, isn't it?]. In "Langsam vermisse ich die Schule... "Schule während und nach der Corona-Pandemie. Edited by Detlef Fickermann and Benjamin Edelstein. Münster: Waxmann, pp. 79-94.

Wanders, Frank H. K., Anne Bert Dijkstra, Ralf Maslowski, and Ineke van der Veen. 2020. The Effect of Teacher-Student and Student-Student Relationships on the Societal Involvement of Students. Research Papers in Education 35: 266-86. [CrossRef]

Wildemann, Anja, and Ingmar Hosenfeld. 2020. Bundesweite Elternbefragung zu Homeschooling während der covid 19-Pandemie: Erkenntnisse zur Umsetzung des Homeschoolings in Deutschland. [Nationwide Parent Survey on Homeschooling during the Covid 19 Pandemic: Insights into the Implementation of Homeschooling in Germany]. Available online: http://www.zepf.eu/ wp-content/uploads/2020/06/Bericht_HOMEschooling2020.pdf (accessed on 22 December 2020).

Xie, Xinyan, Qi Xue, Yu Zhou, Kaiheng Zhu, Qi Liu, Jiajia Zhang, and Ranran Song. 2020. Mental Health Status among Children in Home Confinement during the Coronavirus Disease 2019 Outbreak in Hubei Province, China. JAMA Pediatrics 174: 898-900. [CrossRef] [PubMed] 